\title{
Distributed Link Scheduling for Throughput Maximization under Physical Interference Model
}

\author{
Yaqin Zhou*, Xiang-Yang Li ${ }^{\dagger},+$, Min Liu*, Zhongcheng Li* ${ }^{*}$, Shaojie Tang ${ }^{\dagger}$, Xufei Mao ${ }^{\ddagger}$, and Qiuyuan Huang ${ }^{\S}$ \\ *Institute of Computing Technology, Chinese Academy of Sciences, Beijing, China \\ †Illinois Institute of Technology, Chicago, IL, USA \\ $\ddagger$ TNLIST, School of Software, Tsinghua University, Beijing, China

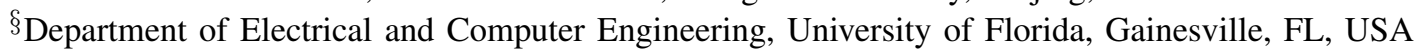

\begin{abstract}
We study distributed link scheduling for throughput maximization in wireless networks. The majority of results on link scheduling assume binary interference models for simplicity. While the physical interference model reflects the physical reality more precisely, the problem becomes notoriously harder under the physical interference model. There have been just a few existing results on centralized link scheduling under the physical interference model, though distributed schedulings are more practical. In this paper, by leveraging the partition and shifting strategies and the pick-and-compare scheme, we present the first distributed link scheduling algorithm that can achieve a constant fraction of the optimal capacity region subject to physical interference constraints in the linear power setting for multihop wireless networks.
\end{abstract}

\section{INTRODUCTION}

As a fundamental problem in wireless networks, link scheduling is crucial for improving networking performances through maximizing throughput and fairness. It has recently regained much interest from networking research community because of wide deployment of multihop wireless networks, e.g., wireless sensor networks for monitoring environment [1] [2] through collection of sensing data [3]. Generally, link scheduling involves determination of which links should transmit at what times, what modulation and coding schemes to use, and at what transmission power levels should communication take place. Despite of its great significance in wireless networks, developing an efficient scheduling algorithm is extremely difficult due to the intrinsically complex interference among simultaneously transmitting links in the network.

The link scheduling problem has been studied in several visions with different optimization objectives, e.g., maximum throughput scheduling, minimum length scheduling. Our study only focuses on the maximum throughput scheduling in multihop wireless networks. Nevertheless, a common primary issue here is to find a set of conflict-free links with regard to the interference models. Thus, interference models matter significantly in link scheduling algorithm design and analysis.

Despite of the numerous significant results [4], [5], [6], [7], [8] to address this problem, most results assume simple binary interference models where a set of links are conflict-free if they are pairwise conflict-free. The predetermined interference relationships can be represented by conflict graphs, and we can leverage classic graph-theoretical tools for solutions. However, in actual wireless communication, interference constraints among concurrent transmissions are not local and pairwise, but global and additive. The determination of a transmission shall consider the cumulative interference from all possible concurrent transmissions, which is often depicted by the physical interference model. However, the simplification of binary interference models from physical reality renders the corresponding results no longer hold in reality. Moreover, the global and additive nature of the interference drives previous traditional techniques based on conflict graphs inapplicable or trivial. Consequently, design and analysis of scheduling algorithms under the physical interference model becomes especially challenging.

Some recent results [9], [10], [11], [12], [13], [14], [15], [16], [17] have addressed a few challenges related to the scheduling problem under the physical interference model. To the best of our knowledge, however, these results, for throughput maximization or other optimization objectives such as a minimum length schedule [10], just focus on centralized implementation. Though results [18], [19] consider distributed implementation of centralized algorithms, unfortunately they fail to provide an exact theoretical guarantee for the achievable performance. Distributed or even localized scheduling under the physical interference model is thus demanding and challenging. One of the main challenges lies in the contradiction that only local information is available to compute a schedule with globally coupled interference constraint.

We tackle these challenges of practical distributed scheduling for throughput maximization under the realistic physical model in this paper. For the primary challenge of decoupling the global interference constraints, on observing that distance dominates the interference, we partition the links into disjoint local link sets with a certain distance away from each other. In this way, the interference from all the other local link sets will be bounded by a constant so that independent scheduling inside each local link set is possible. Thus we can use the partition and shifting strategies to realize an effective distributed implementation. Furthermore, we novelly combine them into the pick-and-compare scheme so that we can prove that our algorithm obtains theoretical capacity guarantee .

We summarize our main contributions as follows. We successfully decouple the global interference constraints. We design a method that will produce disjoint independent sets of links, where the distance between any tow sets of links 
is at least a constant. We show that disjoint local link sets will result in a global independent link scheduling under the linear power setting. We then prove that our distributed link scheduling algorithm under the linear power setting achieves a capacity region that is at least a constant factor of optimum.

The remainder of the paper is organized as follows. In section II, we define the exact system models for the problem we study. In section III, we describe the solution for decoupling the global interference constraints, and the proposed distributed scheduling algorithm with theoretical proof based on it. We conclude this paper in section IV.

\section{Models And Assumptions}

\section{A. Network Communication Model}

We model a wireless network by a graph $G(V, E)$, where $V$ denotes the set of nodes and $E$ denotes the set of links. Each directed link $l=(u, v) \in E$ represents a communication request from a sender $u$ to a receiver $v$. Let $\|l\|$ or $\|u v\|$ denote the length of link $l$. We assume each node knows its own location.

\section{B. Interference model}

Under the physical interference model, a feasible schedule is defined as a set of activated links such that, $S I N R_{u v} \triangleq$ $\frac{p_{u} \cdot \eta \cdot\|u v\|^{-\kappa}}{\sum_{w \in T_{u}} p_{w} \cdot \eta \cdot\|w v\|^{-\kappa}+\xi} \geq \sigma$, where $\xi$ denotes the ambient noise, $\sigma$ denotes certain threshold, and $T_{u}$ denotes the set of simultaneous transmitters with $u$. We assume path gain $\eta \cdot\|u v\|^{-\kappa} \leq 1$, where the constant $\kappa>2$ is path-loss exponent, and $\eta$ is the reference loss factor.

We also assume all links having a length less than the maximum transmission radius $\sqrt[k]{\frac{\eta P}{\sigma \xi}}$, transmit under the linear power setting, where a sender $u$ transmits to a receiver $v$ always at the power $p_{u v}=c \cdot\|u v\|^{\beta} \leq P . c>0$ and $0<\beta \leq \kappa$ are both constants, and $P$ is the maximum transmission power. The distance between $u$ and $v$ satisfies $r \leq\|u v\| \leq R$, where $r$ and $R$ respectively denotes the shortest link length and the longest link length. We suppose that $r$ and $R$ are known by each node.

\section{Traffic models and scheduling}

We also share the same traffic models with [17] [20] where we consider time-slotted wireless systems, and single-hop flows with stationary stochastic packet arrival process at an average arrival rate $\lambda_{l}$. The capacity region, defined as the set of arrival rate vectors $\vec{\lambda}=\left\{\lambda_{1}, \lambda_{2}, \ldots, \lambda_{|E|}\right\}$ under which the system is stable(i.e., all queues are kept finite)., is a major benchmark of throughput performance for link scheduling algorithm . A throughput-optimal scheduling algorithm can achieve the optimal capacity region, while a sub-optimal scheduling policy can just achieves a fraction of the optimal capacity region. The fractionally guaranteed capacity region is depicted by efficiency ratio $\gamma$ [5].

Since a suboptimal scheduling policy with efficiency ratio $\gamma$ [5] must find a $\gamma$-approximation scheduling at every time slot $t$ to achieve $\gamma$ times of the optimal capacity region, it remains difficult to be satisfied in a decentralized scheme. The following called the pick-and-compare is proposed so that we just need to find a $\gamma$-approximation scheduling with a certain constant positive probability.

Proposition 1: ( [8]) Given any $\gamma \in(0,1]$, suppose that an algorithm has a probability at least $\delta>0$ of generating an independent set $\mathcal{X}(t)$ of links with weight at least $\gamma$ times the weight of the optimal. Then, capacity $\gamma \cdot \Lambda$ can be achieved by switching links to the new independent set when its weight is larger than the previous one(otherwise, previous set of links will be kept for scheduling). The algorithm should generate the new scheduling $S(t)$ from the old scheduling $S(t-1)$ and current queue length $Q(t)$.

In the rest of the paper we look for solutions to generate distributed link schedulings, weight of which has a constant approximation ratio to the optimal weight with a certain constant probability at least.

\section{The Algorithm}

In this section, we focus on the design of our algorithm. We firstly demonstrate the basic ideas before involved in the details of implementation.

\section{A. Basic idea}

The basis of our idea is to create a set of disjoint local link sets in which the scheduling can be done independently without violating the global interference constraint. The decoupling of the global interference constraint is based on the fact that distance dominates the interference between two distinct links. That is, if a transmitting link is placed a certain distance away from all the other transmitting links, the total interference it receives may get bounded. We prove later that this assumption holds actually. Based on this, then we employ the partition strategies to divide the network graph into disjoint local areas such that each local area is separated away by a certain distance to enable independent local computation of schedulings inside every local area. Links lying outside local areas will keep silent to ensure separation of local areas. As links lying outside local areas cannot remain unscheduled all the time or it will induce network instability, we use the shifting strategy to change partitions at every time slot to make sure that every backlogged link will be scheduled. These locally computed scheduling link sets compose a new global schedule $\mathcal{X}(t)$ at every time slot $t$.

In light of the pick-and-compare scheme, we choose a more weighted schedule, denoted as $S(t)$, between a new generated schedule $\mathcal{X}(t)$ and the last-time schedule $S(t-1)$ using $Q(t)$. Meanwhile, by Proposition 1, if we guarantee that $\operatorname{Pr}(S(t)$. $\left.Q(t) \geq \gamma S^{*}(t) \cdot Q(t)\right) \geq \delta$ for some constant $\gamma>0, \delta>0$, the queue length vector $Q(t)$ will eventually converge to a stable state if arrival rates vector inside supported region. Then we can get a constant approximation ratio scheduling policy for the optimal.

\section{B. Detailed description}

In this section we describe our solutions detailedly. 
TABLE I

SUMMARY OF NOTATIONS

\begin{tabular}{c|c|c|c|c|c}
\hline$J$ & side length of sub-square & $L_{i j}$ & link set of sub-square $(i, j)$ & $Y_{i j}$ & link set of super-subSquare $(i, j)$ \\
\hline$K$ & side length of super-subSquare & $\mathcal{X}_{i j}(t)$ & new scheduling for $L_{i j}$ at $t$ & $S_{i j}(t)$ & scheduling for $Y_{i j}$ at $t$ \\
\hline$Z_{i}$ & local link set & $O P T_{i j}^{*}(t)$ & local optimal MWISL for $L_{i j}$ & $S^{*}(t)$ & global optimal MWISL at $t$ \\
\hline$R$ & longest link length & $S_{i j}^{*}$ & intersection of $L_{i j}$ and $S^{*}(t)$ & $S(t)$ & global scheduling at time slot (t) \\
\hline$d$ & side length of cell & $\|u v\|$ & link length & $r_{S}(l)$ & relative interference $l$ get from link set $S$ \\
\hline$a_{S}(l)$ & affectness $l$ get from link set $S$ & $Q(t)$ & queue length vector & $W(S)$ & weight of link set $\mathrm{S}$ \\
\hline$I_{S}^{l}$ & interference link $l$ suffered from link set $S$ & $I_{\max }^{l}$ & the maximum interference $l$ can bear & $I_{\max }$ & the minimum of $I_{\max }^{l}$ \\
\hline
\end{tabular}

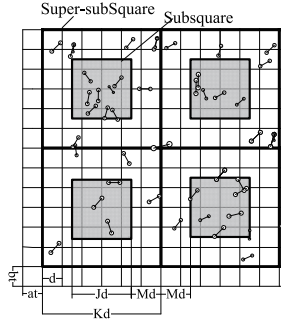

Fig. 1. Partition $\left(K, a_{t}, b_{t}\right)$. Here the gray area is the local area the link set of which participate in computing the new schedule; the links in the white area keep silent.

We first give a brief description of the partition and shifting strategies [20], as illustrated in Fig. 1. We partition the plane into cells with size $d \times d$ where $d=R$, using horizontal lines $x=i$ and vertical lines $y=j$ for all integers $i$ and $j$. A vertical strip with index $i$ is $\{(x, y) \mid i<x \leq i+1\}$. Similarly, we define the horizontal strip $j$. Let cell $(i, j)$ denote the intersection area of a vertical strip $i$ and horizontal strip $j$. A super-subSquare $(i, j)$ is the set of cells: $\{\operatorname{cell}(x, y) \mid x \in$ $\left.\left[i * K+a_{t},(i+1) * K+a_{t}\right), y \in\left[j * K+b_{t},(j+1) * K+b_{t}\right)\right\}$, and a sub-square $(i, j)$ inside it is the set of cells: $\{\operatorname{cell}(x, y) \mid x \in$ $\left[i * K+a_{t}+M,(i+1) * K+a_{t}-M\right), y \in\left[j * K+b_{t}+M,(j+\right.$ $\left.\left.1) * K+b_{t}-M\right)\right\}$. The corresponding link set $Y_{i j}$ (or $L_{i j}$ ) consists of links with both ends inside super-subSquare $(i, j)$ (or sub-square $(i, j)$ ). Let $K=2 M+J, 0 \leq a_{t}, b_{t}<K$. $a_{t}, b_{t}$ are adjustable variables, referred to as the horizontal and vertical shifting respectively. $M$ is a constant distance to keep independence of link scheduling of local areas, which we will formally define later in Lemma 2 . We define the above process as Partition $\left(K, a_{t}, b_{t}\right)$. By adjusting $a_{t}, b_{t}$ separately, we can get $K^{2}$ different partitions for a plane totally.

Then at each time slot nodes cooperate to compute a distributed scheduling. Since every node knows the locality from which it will collect information, it then participates the corresponding local computation, and at last it sends (if it is a coordinator which is closest to the center of the supersubSquare) or receives (if not) the results. The coordinator computes a new local scheduling link set by enumeration in time $n_{i j}^{\Lambda}(t)$ where $n_{i j}(t)$ is the number of nodes in subsquare $(i, j)$, because the size of interference-free links for a sub-square $(i, j)$ is bounded by a constant which we will prove later in Lemma 1. A detailed description is as follows.

Step 1: At time slot 0 , every node first decides in which cell it resides by a partition using $\left(a_{0}, b_{0}\right)=(0,0)$; then it participates in the computation of the corresponding local scheduling $\mathcal{X}_{i j}(0)$. Let the solution $S(0)$ of time slot 0 be the union of the local solutions $\mathcal{X}_{i j}(0)$ from all sub-squares.

Step $m+1$ : For any time-slot $t, t>0$, every node decides in which cell it resides by a partition starting from $\left(a_{t}, b_{t}\right)$. For better illustration we let $a_{t}=t \bmod K$, and $b_{t}=\left(b_{t}+1\right)$ $\bmod K$ if $a_{t}=0, b_{t}$ keeps unchanged otherwise. Each node then participates in computing the new local scheduling, denoted as $\mathcal{X}_{i j}(t)$, for its sub-square $(i, j)$ using the weight $Q(t)$. Let $S_{i j}(t-1)$ be the set of links from $S(t-1)$ (the global solution at time slot $t-1)$ falling in the super-subSquare $(i, j)$ instead of sub-square $(i, j)$. If $S_{i j}(t-1) \cdot Q(t)>\mathcal{X}_{i j}(t) \cdot Q(t)$, let $S_{i j}(t)=S_{i j}(t-1)$, else $S_{i j}(t)=\mathcal{X}_{i j}(t)$, the global solution is the union of $S_{i j}(t)$ from all super-subSquares.

\section{Theoretical analysis and proof}

We then prove that our algorithm is correct and has a constant approximation ratio to the optimal capacity region.

Given a network $G(V, E)$, supposing $\cup Z_{i}$ is a set of disjoint local link sets inside for scheduling, where $Z_{i} \in E$ and $Z_{i} \cap$ $Z_{j}=\phi$ if $i \neq j$, for any link $l \in Z_{i}$, if $l$ is activated, then

$$
I_{l, l \in Z_{i}}=I_{\text {in }}^{l}+I_{\text {out }}^{l}
$$

where $I_{l, l \in Z_{i}}$ denotes cumulative interference from all other activated links in the network, $I_{i n}^{l}$ denotes the total interference from simultaneously transmitting links inside $Z_{i}, I_{\text {out }}^{l}$ denotes the total interference from transmissions outside.

Therefore, we can do independent scheduling inside $Z_{i}$ without consideration of $I_{\text {out }}^{l}$ from concurrent transmissions outside $Z_{i}$, if $I_{\text {out }}^{l}$ gets bounded by a constant, i.e.,

$$
I_{\text {in }}^{l} \leq(1-\varepsilon) \cdot I_{\max }^{l}, I_{\text {out }}^{l} \leq \varepsilon \cdot I_{\max }, 0<\varepsilon<1, l \in Z_{i} .
$$

$I_{\max }$ is the maximum interference that the longest links in $E$ can tolerant during a successful transmission and $I_{\max }^{l}$ represents the maximum interference that $l$ can tolerant during a successful transmission.

We will give a formal statement that $I_{\text {out }}^{l}$ is indeed bounded by $\varepsilon I_{\max }$ in Lemma 2 . However, to cooperate proof of Lemma 2 , we shall claim the following Lemma 1 in advance.

Lemma 1: In the linear power setting, the number of interference-free links for a local link set $Z_{i}$ inside a square with a side length $J R$ is bounded by a constant. Let $O P T_{i}$ refer to the optimal MWISL for $Z_{i}$. That is,

$$
\left|O P T_{i}\right| \leq \frac{(\sqrt{2} J R)^{\kappa}}{(1-\varepsilon)}\left[\frac{1}{\sigma}-\frac{\xi \cdot\left\|l^{*}\right\|^{\beta-\kappa}}{c \eta}\right]+1 .
$$

Proof: The proof is available in Appendix A.

We let $\left|O P T_{i}\right|_{u b}$ denote an upper bound of the size of the local optimal MWISL for $Z_{i}$, then we have,

Lemma 2: In a given network $G(V, E)$ under the physical interference model in the linear power setting, if the Euclidean distance between any two disjoint local link sets is at least $M \times R$, then activated links in each local link set suffer a bounded cumulative interference from all other activated link sets, i.e., for each activated link $l$ in local link set $Z_{i}$,

$$
I_{\text {out }}^{l} \leq \varepsilon \cdot I_{\max }, 0<\varepsilon<1,
$$

$I_{o u t}^{l} \leq \varepsilon \cdot I_{\max }, 0<\varepsilon<1$,
where $\mathrm{M}$ is a constant, satisfying $M \geq\left[\frac{2 \pi c \eta R^{\beta-\kappa} \cdot\left|O P T_{i}\right|_{u b}}{(\kappa-2) \varepsilon I_{\max }}\right]^{\frac{1}{\kappa}}$. 
Proof: The proof is available in Appendix B.

To analyze the theoretical performance of our method, we first review the following definitions.

Definition 2: (affectness [14]) The relative interference of link $l_{w}$ on $l_{v}$ is the increase caused by $l_{w}$ in the inverse of the SINR at $l_{v}$, namely $r_{l_{w}}\left(l_{v}\right)=I_{l_{w}}^{l_{v}} / P_{l_{v}}$. For convenience, define $r_{l_{v}}\left(l_{v}\right)=0$. Let $c_{v}=\frac{\sigma}{1-\sigma \xi / P_{l_{v}}}$ be a constant that indicates the extent to which the ambient noise approaches the required signal at receiver $t_{v}$. The affectness of link $l_{v}$ caused by a set $S$ of links, is the sum of relative interference of the links in $S$ on $l_{v}$, scaled by $c_{v}$, or $a_{S}\left(l_{v}\right)=c_{v} \cdot \sum_{l_{w} \in S} r_{l_{w}}\left(l_{v}\right)$.

Definition 3: (p-signal set [14]) We define a $p$-signal set to be one where the affectness of any link is at most $1 / p$. Clearly, any ISL is a 1-signal set.

Lemma 3: ( [14]) There is a polynomial-time protocol that takes a $p$-signal set and refines into a $p^{\prime}$-signal set, for $p^{\prime}>p$, increasing the number of slots by a factor of at most $4\left(\frac{p^{\prime}}{p}\right)^{2}$.

Next in Lemma 4 we exposit a constant approximation relationship between each locally computed scheduling link set and its counterpart of the global optimal scheduling set.

Lemma 4: The weight of $\mathcal{X}_{i j}(t)$ has a constant approximation ratio to the weight of the intersection set by the local link set $L_{i j}$ and the global optimal MWISL $S^{*}(t)$.

Proof: Normally any ISL is a 1-signal set. Nevertheless, in order to keep independence of sub-squares, the locally computed ISL should be a $p$-signal set, where $p$ is bigger than 1 . Thus the affectness of a locally computed ISL for subsquare must satisfy that,

$a_{S_{i j}}\left(l_{v}\right)=c_{v} \cdot \sum_{l_{w} \in S_{i j}} r_{l_{w}}\left(l_{v}\right) \leq \frac{\sigma}{1-\sigma \xi / P_{l_{v}}} \cdot(1-\varepsilon) \cdot \frac{I_{l_{v}}^{\max }}{P_{l_{v}}} \leq 1-\varepsilon$.

Therefore, by Lemma 3, any $p^{\prime}$-signal set can be refined into at most $4\left(\frac{p^{\prime}}{p}\right)^{2} p$-signal sets, where $p^{\prime}>p$. So a normal MWISL can be refined into $\frac{4}{(1-\varepsilon)^{2}} \frac{1}{(1-\varepsilon)}$-Signal link sets at most. Since the $\frac{1}{(1-\varepsilon)}$-Signal link set returned by enumeration is most weighted, so the locally computed link scheduling sets $\mathcal{X}_{i j}(t)$ has $W\left(\mathcal{X}_{i j}(t)\right) \geq \frac{(1-\varepsilon)^{2}}{4} W\left(O P T_{i j}(t)\right)$.

Let $S_{i j}^{*}(t)=S^{*}(t) \cap L_{i j}$ denote the intersection by $L_{i j}$ and $S^{*}(t)$, where $S^{*}(t)$ is the global optimal MWISL at time slot $t$. Let $W(S)$ denote the sum weight of links in a link set $S$. It is obvious that $W\left(S_{i j}^{*}(t)\right) \leq W\left(O P T_{i j}(t)\right) \leq \frac{4}{(1-\varepsilon)^{2}} W\left(\mathcal{X}_{i j}(t)\right)$.

Thus it proves the lemma.

Theorem 1: $S(t)=\cup S_{i j}(t)$ computed by our algorithm is an independent link set under the physical interference model in the linear power setting. The weight of $S(t), W(S(t))$, is a constant approximation of the weight of the global optimal MWISL with probability at least $1 / K^{2}$.

Proof: We prove the theorem in two phases. We first prove that $S(t)=\cup S_{i j}(t)$ is an independent set. We then prove the approximation bound $S(t)$ achieves.

For any link $l \in S(t)$, assuming $l \in S_{i j}(t)$, the total interference $l$ suffers from all the other simultaneously transmitting links in $S(t)$ is denoted by $I_{S(t)}^{l}$.

At time slot 0 , every local activated link set $S_{i j}(0)=\mathcal{X}_{i j}(0)$ is kept $2 M$ cells away from each other, so $S(0)$ is an independent set by Lemma 2 .
At time slot 1 , either $S_{i j}(0)$ or $\mathcal{X}_{i j}(1)$ is chosen to be a part of $S(1)$. For those super-subSquares whose $S_{i j}(1)=$ $S_{i^{\prime} j^{\prime}}(0) \cap Y_{i j}(0)$, their distance is kept at least $2 M$ cells away. For those super-subSquares whose $S_{i j}(1)=\mathcal{X}_{i j}(1)$, their distance is also kept at least $2 M$ cells away. And he distance between the two kinds of link set is at least $2 M-1$ cells away. So $S(1)$ is an independent set.

At some time slot $t^{*}, t^{*}>1$, for some super-subSquares, $S_{i j}\left(t^{*}\right)$ consists of disjoint subsets from several different $S_{i^{\prime} j^{\prime}}\left(t^{*}-1\right)$ which fall into $Y_{i j}\left(t^{*}\right)$, i.e., $S_{i j}\left(t^{*}\right)=S\left(t^{*}-1\right) \cap$ $Y_{i j}\left(t^{*}\right)=\bigcup_{i^{\prime} j^{\prime}}\left\{S_{i^{\prime} j^{\prime}}\left(t^{*}-1\right) \cap Y_{i j}\left(t^{*}\right)\right\}$. We then let $\Phi_{i^{\prime} j^{\prime}}^{i j}\left(t^{*}\right)=$ $S_{i^{\prime} j^{\prime}}\left(t^{*}-1\right) \cap Y_{i j}\left(t^{*}\right)$ for brevity. Since each $S_{i^{\prime} j^{\prime}}\left(t^{*}-1\right)$ is kept at least $M$ cells away from each other, so is each $\Phi_{i^{\prime} j^{\prime}}^{i j}$.

Clearly, $S\left(t^{*}\right)$ can be divided into two separated subsets, one formed by some subsets of $S\left(t^{*}-1\right)$, the other formed by newly computed $\mathcal{X}_{i j}\left(t^{*}\right)$, i.e.,

$$
S\left(t^{*}\right)=\bigcup_{i j} S_{i j}\left(t^{*}\right)=\left\{\bigcup_{p q} \bigcup_{i^{\prime} j^{\prime}} \Phi_{i^{\prime} j^{\prime}}^{p q}\left(t^{*}\right)\right\} \bigcup\left\{\bigcup_{\substack{m n, m n \neq p q}} \mathcal{X}_{m n}\left(t^{*}\right)\right\} .
$$

Since $\bigcup_{p q} \bigcup_{i^{\prime} j^{\prime}} \Phi_{i^{\prime} j^{\prime}}^{p q}\left(t^{*}\right)$ is a subset of $S\left(t^{*}-1\right)$, it is composed by disjoint subsets with a mutual distance of $M$ cells at least. The distance between any distinct $\mathcal{X}_{m n}\left(t^{*}\right)$ is no less than $2 M$ cells. Then we consider the distance between a disjoint subset of $\bigcup_{p q} \bigcup_{i^{\prime} j^{\prime}} \Phi_{i^{\prime} j^{\prime}}^{p q}\left(t^{*}\right)$ and a $\mathcal{X}_{m n}\left(t^{*}\right)$. Since $\mathcal{X}_{m n}\left(t^{*}\right)$ locates in sub-square $(m, n)$, which is $M$ cells away from the border of super-subSquare $(m, n)$, the distance between a disjoint subset of $\bigcup_{p q} \bigcup_{i^{\prime} j^{\prime}} \Phi_{i^{\prime} j^{\prime}}^{p q}\left(t^{*}\right)$ and a $\mathcal{X}_{m n}\left(t^{*}\right)$ is still no less than $M$ cells. Comprehensively, $S\left(t^{*}\right)$ consists of disjoint subsets which are separated by at least $M$ cells.

Note that a disjoint subset of $S\left(t^{*}\right)$ does not equalize to a $S_{i j}\left(t^{*}\right)$ since a $S_{i^{\prime} j^{\prime}}\left(t^{*}-1\right)$ may be reserved completely in different super-subSquares at time slot $t^{*}$. Here we denote the disjoint subset by $\psi_{i}\left(t^{*}\right)$, and $S\left(t^{*}\right)=\bigcup \psi_{i}\left(t^{*}\right)$.

By Lemma 2, for each link $l \in \psi_{i}\left(t^{*}\right)$, where $\psi_{i}\left(t^{*}\right)$ comes from $\bigcup_{p q} \bigcup_{i^{\prime} j^{\prime}} \Phi_{i^{\prime} j^{\prime}}^{p q}\left(t^{*}\right)$, we have $I_{S\left(t^{*}\right)}^{l} \leq I_{\max }^{l}$. Meanwhile, for each $l \in \psi_{i}\left(t^{*}\right)$, where $l \in \psi_{i}\left(t^{*}\right)$ comes from $\bigcup \mathcal{X}_{m n}\left(t^{*}\right)$, it holds that $I_{S\left(t^{*}\right)}^{l} \leq I_{\max }^{l}$. Then for any $m n, m n \neq p q$

$l \in S\left(t^{*}\right), I_{S\left(t^{*}\right)}^{l} \leq I_{\max }^{l}$, thus $S\left(t^{*}\right)$ is an independent set.

Next we consider situations at time slot $t^{*}+1$. Similarly, $S\left(t^{*}+1\right)$ composes of disjoint subsets separated by no less than $M$ cells. Using the same technique as at time slot $t^{*}$, we can get that $S\left(t^{*}+1\right)$ is still an independent set.

By induction we can infer that $S(t)$ is a union of disjoint activated subsets separated by $M$ cells at least, thus it is an independent. Herein we finish the first phrase of the proof.

The next is the proof of the second phrase.

Note that there are $K^{2}$ different partitions for a plane totally. So the partition $\left(K, a_{t}, b_{t}\right)$ has a probability at least $1 / K^{2}$ to be an optimal partition, the weight of whose corresponding $S(t)$ is an upper bound of that of any other partition. Observing that each cell $(i, j)$ appears in the "removed" strips at most $2 \mathrm{KM}$ times during $K^{2}$ different partitions. Let $D(t)$ denote 
the link set of the removed strips at Partition $\left(a_{t}, b_{t}, K\right) . D^{*}(t)$ represents the subset of $S^{*}(t)$, links of which fall inside $D(t)$, i.e., $D^{*}(t)=D(t) \cap S^{*}(t)$. For an optimal partition it satisfies that, $W\left(D_{i}^{*}(t)\right) \leq \frac{2 K M}{K^{2}} \cdot \sum_{i=0}^{K^{2}-1} W\left(D_{i}^{*}(t)\right)=\frac{2 M}{K} W\left(S^{*}(t)\right)$, and $W\left(\cup S_{i j}^{*}(t)\right)=W\left(S^{*}(t) \backslash D^{*}(t)\right) \geq\left(1-\frac{2 M}{K}\right) W\left(S^{*}(t)\right)$. So by Lemma 4, we can get $\left(1-\frac{2 M}{K}\right) W\left(S^{*}(t)\right) \leq \frac{4}{(1-\varepsilon)^{2}} W\left(\cup \mathcal{X}_{i j}(t)\right)$.

By Proposition 1 we derive that the approximation ratio for the optimal is $\frac{4}{\left(1-2 \cdot \frac{M}{K}\right)(1-\varepsilon)^{2}}$ This finishes the proof.

\section{CONCLUSION}

We tackle the problem of distributed link scheduling for throughput maximization in wireless networks subject to physical interference with the linear power assignment. We successfully address the primary challenge of global interference constraint that hinders development of distributed scheduling algorithms. We then utilize the graph partition and shifting techniques to achieve distributed schedulings with a constant approximation ratio to the optimal solution.

Acknowledgements: The research of authors is partially supported by NSF CNS-0832120, NSF CNS-1035894, National Natural Science Foundation of China under Grant No. 61170216, No.61132001, No. 61120106008, No. 61070187, No. 60970133, No. 61003225, National Basic Research Program of China (973 Program) under grant No. 2010CB328100, 2010CB334707, 2011CB302705, 2011CB302702, Tsinghua National Laboratory for Information Science and Technology (TNList), program for Zhejiang Provincial Key Innovative Research Team, program for Zhejiang Provincial Overseas High-Level Talents (One-hundred Talents Program), and the Beijing Nova Program.

\section{REFERENCES}

[1] M. Li and Y. Liu, "Underground coal mine monitoring with wireless sensor networks," ACM Transactions on Sensor Networks (TOSN), vol. 5, no. 2, p. 10, 2009.

[2] Y. Liu, K. Liu, and M. Li, "Passive diagnosis for wireless sensor networks," IEEE/ACM Transactions on Networking (TON), vol. 18, no. 4, pp. 1132-1144, 2010.

[3] X.-H. Xu, X.-Y. Li, P.-J. Wan, and S.-J. Tang, "Efficient scheduling for periodic aggregation queries in multihop sensor networks," IEEE/ACM Transactions on networking, Aug. 2011.

[4] L. Tassiulas and A. Ephremides, "Linear complexity algorithms for maximum throughput in radio networks and input queued switches," in Proc. IEEE Infocom, 1998, p. 533.

[5] C. Joo, X. Lin, and N. B. Shroff, "Understanding the capacity region of the greedy maximal scheduling algorithm in multi-hop wireless networks," in Proc. IEEE Infocom, 2008, pp. 1103-1111.

[6] X. Lin and S. B. Rasool, "Constant-time distributed scheduling policies for ad hoc wireless networks," IEEE/ACM Transactions on Automatic Control, vol. 54, pp. 231-242, Feb. 2009.

[7] A. Gupta, X. Lin, and R. Srikant, "Low-complexity distributed scheduling algorithms for wireless networks," IEEE/ACM Transactions on Networking, vol. 17, pp. 1846-1859, Dec. 2009.

[8] S. Sanghavi, L. Bui, and R. Srikant, "Distributed link scheduling with constant overhead," in Proc. ACM SIGMETRICS, 2007, pp. 313-324.

[9] O. Goussevskaia, Y. Oswald, and R. Wattenhofer, "Complexity in geometric SINR," in Proc. ACM Mobihoc, 2007, pp. 100-109.

[10] P.-J. Wan, O. Frieder, X.-H. Jia, F. Yao, X.-H. Xu, and S.-J. Tang, "Wireless link scheduling under physical interference model," in Proc. IEEE Infocom, 2011, p. 838.

[11] O. Goussevskaia, R. Wattenhofer, M. M. Halldorsson, and E. Welzl, "Capacity of arbitrary wireless networks," in Proc. IEEE Inforcom, 2009, pp. $1872-1880$
[12] X.-Y. Li, "Multicast capacity of wireless ad hoc networks," IEEE/ACM Transactions on networking, vol. 17, pp. 950-961, June 2009.

[13] X.-H. Xu, S.-J. Tang, and P.-J. Wan, "Maximum weighted independent set of links under physical interference model," in LNCS, vol. 6221. Springer, Heidelberg, 2010, pp. 68-74.

[14] M. Halldorsson and R. Wattenhofer, "Wireless communication is in APX," in Proc. 36th International Colloquium on Automata, Languages and Programming, 2009.

[15] D. Chafekar, V. Kumar, M. Marathe, S. Parthasarathy, and A. Srinivasan, "Arrpoximation algorithms for computing capacity of wireless networks with SINR constraints," in Proc. IEEE Infocom, 2008, pp. 1166-1174.

[16] P.-J. Wan, X.-H. Xu, and O. Frieder, "Shortest link scheduling with power control under physical interference model," in Proc. IEEE MSN, 2010, pp. 74-78.

[17] X. H. Xu, S. J. Tang, and X.-Y. Li, Stable Wireless Link Scheduling Subject to Physical Interferences With Power Control, 2011, manuscript.

[18] D. M. blough, G. Resta, and P. Santi, "Approximation algorithms for wireless link scheduling with SINR-Based inteference," IEEE/ACM Transactions on networking, vol. 18, pp. 1701-1712, Dec. 2010.

[19] L.-B. Le, E. Modiano, C. Joo, and N. B. Shroff, "Longest-queue-first scheduling under SINR interference model," in Proc. ACM Mobihoc, 2010, pp. 41-50.

[20] S.-J. Tang, X.-Y. Li, X. Wu, Y. Wu, X. Mao, P. Xu, and G. Chen, "low complexity stable link scheduling for maximizing throughput in wireless networks," in Proc. IEEE SECON, 2009, pp. 1-9.

\section{APPENDIX A}

PROOF OF LEMMA 1

Proof: Suppose $l^{*}$ is the shortest link in $O P T_{i}$, let $l$ denote any other link different from $l^{*}$ in $O P T_{i}$. Assuming $l^{*}=\left(u^{*}, v^{*}\right), l=(u, v)$, we have $\left\|u v^{*}\right\| \leq \sqrt{2} J R$. The relative interference $l^{*}$ received from $O P T_{i}$ is shown below.

$$
\begin{aligned}
r_{O P T_{i}}\left(l^{*}\right) & =\frac{l \in\left\{O P T_{i} \backslash\left\{l^{*}\right\}\right\}}{c \cdot\|l\|^{\beta} \cdot \eta \cdot\left\|u v^{*}\right\|^{-\kappa}} \\
& \geq \sum_{l \in\left\{O P T_{i} \backslash\left\{l^{*}\right\}\right\}}(\sqrt{2} J R)^{-\kappa} \frac{\|l\|^{\beta}}{\left\|l^{*}\right\|^{\beta}} \\
& \geq\left|O P T_{i}-1\right| \cdot(\sqrt{2} J R)^{-\kappa} .
\end{aligned}
$$

Since $l^{*} \in O P T_{i}$, so it should satisfy the SINR constraint that $\frac{c \cdot\left\|l^{*}\right\|^{\beta} \cdot \eta \cdot\left\|l^{*}\right\|^{-\kappa}}{\xi+(1-\varepsilon) I_{\max }^{l *}} \geq \sigma$, then we have

$$
r_{O P T_{i}}^{\max }\left(l^{*}\right) \leq \frac{1}{1-\varepsilon}\left[\frac{1}{\sigma}-\frac{\xi \cdot\left\|l^{*}\right\|^{\kappa-\beta}}{c \eta}\right] .
$$

Therefore we derive $\left|O P T_{i}\right| \leq \frac{(\sqrt{2} J R)^{\kappa}}{1-\varepsilon}\left[\frac{1}{\sigma}-\frac{\xi \cdot r^{\kappa-\beta}}{c \eta}\right]+1$.

\section{APPENDIX B}

\section{PROOF OF LEMMA 2}

Proof: For any two links $l^{*}$ and $l$ which do not belong to the same local link set $Z_{i}$, assuming $l^{*}=\left(u^{*}, v^{*}\right), l=(u, v)$, it always holds that $\left\|u v^{*}\right\| \geq M \times R$.

The total interference $l^{*}$ suffered form all concurrent transmitting links located in other sub-squares is

$$
\begin{aligned}
I_{\text {out }}^{l^{*}} & =\sum_{l \in\left\{\cup Z_{i} \backslash Z^{\prime}\right\}, l^{*} \in Z^{\prime}} c \times\|l\|^{\beta} \times \eta \times\left\|u v^{*}\right\|^{-\kappa} \\
& \leq c \times \eta \times R^{\beta} \times \sum_{l \in\left\{\cup Z_{i} \backslash Z^{\prime}\right\}, l^{*} \in Z^{\prime}}\left\|u v^{*}\right\|^{-\kappa} \\
& \leq c \times \eta \times R^{\beta} \times \int_{1}^{\infty} \frac{2 \pi x M R}{M R} \times\left|O P T_{i}\right|_{u b} \times(x M R)^{-\kappa} d x \\
& =c \times \eta \times R^{\beta-\kappa} \times 2 \pi \times\left|O P T_{i}\right|_{u b} \times M^{-\kappa} \times \frac{1}{\kappa-2}
\end{aligned}
$$

since $\kappa$ is a constant greater than 2 typically.

To assure $I_{\text {out }}^{l^{*}} \leq \varepsilon \cdot I_{\max }$, we derive that

$$
M \geq\left[\frac{2 \pi c \eta R^{\beta-\kappa} \cdot\left|O P T_{i}\right|_{u b}}{(\kappa-2) \varepsilon I_{\max }}\right]^{\frac{1}{\kappa}}
$$

where $I_{\max }=\frac{c \eta R^{\beta-\kappa}}{\sigma}-\xi$ is a constant for a given network $G,\left|O P T_{i}\right|_{u b}$ is a constant upper bound of $\left|O P T_{i}\right|$. 